\title{
Relationship between depression and medication adherence in cardiovascular disease: the perfect challenge for the integrated care team
}

This article was published in the following Dove Press journal:

Patient Preference and Adherence

15 March 2017

Number of times this article has been viewed

\author{
Carly M Goldstein ${ }^{1,2}$ \\ Emily C Gathright ${ }^{1,3}$ \\ Sarah Garcia ${ }^{4}$ \\ 'Department of Psychiatry \\ and Human Behavior, Warren \\ Alpert Medical School of Brown \\ University, ${ }^{2}$ Weight Control and \\ Diabetes Research Center, The \\ Miriam Hospital, Providence, RI, \\ ${ }^{3}$ Department of Psychological \\ Sciences, Kent State University, Kent, \\ $\mathrm{OH},{ }^{4}$ Neuropsychology Section, \\ Department of Psychiatry, University \\ of Michigan, Ann Arbor, MI, USA
}

\begin{abstract}
Many individuals with cardiovascular disease (CVD) experience depression that is associated with poor health outcomes, which may be because of medication nonadherence. Several factors influence medication adherence and likely influence the relationship between depression and medication adherence in CVD patients. This comprehensive study reviews the existing literature on depression and medication adherence in CVD patients, addresses the methods of and problems with measuring medication adherence, and explains why the integrated care team is uniquely situated to improve the outcomes in depressed CVD patients. This paper also explores how the team can collaboratively target depressive symptoms and medication-taking behavior in routine clinical care. Finally, it suggests the limitations to the integrated care approach, identifies targets for future research, and discusses the implications for CVD patients and their families.
\end{abstract}

Keywords: cardiovascular disease, medication adherence, self-management, electronic monitoring, integrated care, collaborative care, compliance

\section{Introduction}

Cardiovascular disease (CVD) is the leading cause of death for American adults, and 85.6 million individuals in the United States are estimated to have one or more types of CVD. ${ }^{1}$ It is also the leading source for US health care expenditures. Much of this health care expense stems from rehospitalization, which frequently follows failure to properly self-manage one's CVD. ${ }^{1}$ In addition, many individuals with CVD experience depression, ${ }^{2}$ a condition that is associated with poorer outcomes in CVD patients. ${ }^{3}$ Medication nonadherence may help explain why depressed CVD patients experience poor outcomes; both depressed and nondepressed CVD patients are at risk of poor medication adherence. ${ }^{4}$

\section{CVD and depression}

There are strong associations between depressive symptoms and CVD. Estimates of depression in CVD vary depending on the specific disease processes and assessment method, but $\sim 50 \%$ of patients experience major or minor depression at least once in the course of experiencing CVD. ${ }^{5}$ In a study that examined individuals hospitalized for myocardial infarction (MI), $\sim 20 \%$ met the criteria for a major depressive episode based on a structured interview, and $31.1 \%$ self-reported significant depressive symptoms. ${ }^{6}$ Individuals with heart failure (HF) seem to display a slightly increased prevalence over one third - particularly when screened through self-report measures. ${ }^{7,8}$ Mechanisms 
for the relationship of depression in CVD are unknown but may include autonomic imbalance, hypercoagulability, endothelial and vascular effects in women, inflammation, and indirect causation from behavior and nonadherence. ${ }^{9}$ Independent predictors of depressive symptoms assessed in the hospital and 1 year post-discharge after MI include pre-MI vital exhaustion, history of depressive disorder, history of MI, poor ejection fraction, longer hospital stay, living alone, poor exercise tolerance, and female gender. ${ }^{10}$

\section{Depression and hospitalization or rehospitalization}

Across CVD patients, depression is associated with increased health service use - depression predicts repeated HF hospitalizations ${ }^{11,12}$ and rehospitalization after acute MI. ${ }^{13}$ For example, acute MI patients with depressive symptoms experienced more emergency department visits and hospitalizations, were hospitalized sooner, and were hospitalized longer than acute MI patients without depression. ${ }^{13,14}$ Similarly, the Heart Failure Adherence and Retention Trial examined whether an intervention of self-management skills and HF-specific education reduced a patient's likelihood of future hospitalizations. ${ }^{11}$ Although the intervention had no effect on the primary endpoint of death or hospitalization for HF compared to HF-specific education alone, ${ }^{15}$ depressed individuals demonstrated 1.45 times more HF-related hospitalizations than nondepressed patients even after adjusting for physician adherence to evidence-based medication use, patient adherence to HF medications and salt restriction, illness severity, HF severity, and socioeconomic factors. ${ }^{11}$

\section{Depression and mortality}

The presence of depressive symptoms in patients with CVD predicts increased risk of death. ${ }^{3,16-18}$ Major depression is associated with increased mortality 3 and 12 months following hospitalization for $\mathrm{CVD}^{19}$ and 1 year after initial assessment in patients with HF. ${ }^{20}$ However, despite decades of research, the scientific community does not fully understand the relationship between depression and mortality. ${ }^{21}$

Initiating treatment for depression through psychotherapy and pharmacotherapy does not eliminate the relationship between depression and mortality in CVD. In one study (Enhancing Recovery in Coronary Heart Disease Patients), researchers discovered that patients who experienced their first major post-MI depressive episode and individuals who experienced a recurrent episode upon MI both had significantly poorer survival rates than did nondepressed patients. ${ }^{22}$ The researchers treated depression through cognitive behavioral therapy (CBT) and a selective serotonin reuptake inhibitor (SSRI) when CBT alone did not significantly reduce depressive symptoms after 5 weeks. ${ }^{23}$ Upon 5-year follow-up, individuals experiencing major or minor depression were at higher risk for all-cause mortality compared with individuals who were not depressed at follow-up. ${ }^{24}$ Antidepressants do not eliminate the relationship between depressive symptoms and mortality in patients with HF. ${ }^{25}$ Depression seems to affect the overall outcomes even when individuals seek treatment, suggesting that depression can powerfully influence the outcomes, and further studies are required to analyze the mechanism for this influence.

In another trial (the Sertraline Antidepressant Heart Attack Randomized Trial), researchers tested the safety and efficacy of sertraline in treating post-MI depression. ${ }^{26}$ Sertraline seemed to work best in participants with a history of depression. Nevertheless, baseline depression severity and failure to improve depression substantially during treatment with either sertraline or placebo were strongly and independently associated with long-term mortality. ${ }^{26}$ In additional studies, insufficiently treated depression and treatment-resistant depression following MI were associated with mortality after controlling for $\beta$-blocker use, sociodemographics, anxiety disorders, mortality factors, and health service utilization compared with treated patients. ${ }^{27}$ Although previous research for decades has sought to improve the community's understanding of depression and consequently decrease mortality, the research findings to date are discouraging - this complex relationship seems to require a much more nuanced perspective.

In general, persistent depressive symptoms are associated with poor adherence across various health behaviors including quitting smoking and attending cardiac rehabilitation, and a patient's failure to adhere to these important behaviors is associated with increased mortality. ${ }^{4} \mathrm{~A}$ multifaceted treatment approach that targets cardiovascular and psychological processes is necessary at minimum, and more robust treatment is likely needed. Not only does depression contribute to poor outcomes, but it also contributes to poor medication adherence, a likely moderator in the relationship between depression and death in CVD.

\section{CVD and medication adherence}

Medication adherence is the extent to which a patient follows his or her provider's recommendations regarding day-to-day treatment such as medication timing, dosage, and frequency. ${ }^{28}$ Medication adherence in CVD patients is often sub-optimal. ${ }^{29}$ Rarely, in routine clinical practice, 
the clinician measures or targets medication adherence. ${ }^{30}$ Following hospital discharge, adherence to cardiovascular medications (eg, statins and $\beta$-blockers) seems to steadily decrease. ${ }^{30}$ Within HF, estimates of medication adherence range from $40 \%$ to $60 \%$, although previous estimates have ranged from $10 \%$ to $93 \%{ }^{31}$

\section{Factors influencing medication adherence in CVD}

The factors contributing to medication nonadherence in CVD are not well established. ${ }^{32}$ The World Health Organization notes that nonadherence can be preventable (eg, a patient forgetting to take his or her medicine) or nonpreventable (eg, life-threatening adverse effects), and health care systems, medical conditions, patients, the therapy itself, and socioeconomic factors can all contribute to medication nonadherence. ${ }^{33}$ Nonadherence in CVD is associated with disease-related complications, hospitalizations, disability, and increased mortality. ${ }^{34}$ In a sample of HF patients using the medication event monitoring system (MEMS) device, lapses in attention, excessive daytime sleepiness, and two or more daily medication doses predicted steep declines in objectively measured medication adherence. ${ }^{35}$ Likewise, New York Heart Association class III patients, individuals with asthma, and individuals with renal disease have been found less likely to adhere to an evidence-based HF medication regimen as assessed by MEMS than those with less severe disease. ${ }^{36}$

Previous research may have underestimated the complexity of adherence behavior. For instance, Brown et al's recent model is one of the first to fully recognize some potential determinants of medication adherence behavior. ${ }^{37}$ In this conceptual model of atrial fibrillation, patients' adherence to oral anticoagulants and components include the following: predisposing, moderating, and contextual factors; knowledge base and reinforcement; short- and long-term motivation; personalized system, habit formation, and system adaptation; and a self-efficacy loop. ${ }^{37}$ This model encompasses many factors previously considered separately in theoretical models of medication adherence and targeted individually in interventions designed to improve medication adherence.

\section{Medication adherence and mortality}

Medication nonadherence may lead to rehospitalization or death. ${ }^{34}$ Among surveyed patients, caregivers, cardiologists, and nurses, respondents noted that better medication adherence likely could have prevented nearly one third of HF readmissions. ${ }^{38} \mathrm{Wu}$ et al aimed to determine the indicators of medication adherence that predict event-free survival using MEMS and self-report. ${ }^{39}$ The MEMS system assessed several factors, namely the percentage of prescribed doses taken (dose-count); the percentage of days when the correct number of doses were taken (dose-days); and the percentage of doses taken on schedule (dose-time). ${ }^{39}$ Dose-count and dose-days - but not self-reported adherence - predicted event-free survival. ${ }^{39}$ Follow-up analyses determined that the five most commonly cited barriers in the study were cost, "forgetting the time of medication," "not carrying the medication when I am out," "amount of pills that I need to take that day," and "belief that I'll be fine even though I skip one dose of medication." ${ }^{40}$ Of note, the discrepancy between self-reported versus objective adherence highlights that the types of measurement used within research have serious implications for what is already known about medication adherence, and outcomes of certain levels of medication adherence, in CVD.

\section{Measurement of medication adherence \\ Methods of measurement}

Adherence methods are either direct or indirect. Direct methods include directly (visually) observed therapy or measurement of a biological marker, level of medicine, or metabolite in the blood. ${ }^{41}$ These methods have limited practicality within routine clinical use. ${ }^{30}$ Indirect methods include patient questionnaires, pill counts, verbal self-reports, electronic medication monitors, measurement of patient diaries, rate of prescription refills, and assessment of the patient's clinical response. ${ }^{41}$

Most indirect measurement types have drawbacks. For example, although pill counts are easy to perform, timing of medication administration is not captured, and hence, the results are easily manipulated. ${ }^{30}$ Electronic pharmacy data (eg, tracking individuals to determine whether they procure their prescriptions) require individuals to obtain medications from a closed pharmacy system, which however fail to capture dose timing. ${ }^{30}$ Self-report measures are a convenient alternative, but can be biased by social desirability. ${ }^{30}$

Many researchers justify using self-report adherence because previous studies have found self-reported adherence to be predictive. ${ }^{42}$ It tends to have high specificity, but low sensitivity. ${ }^{43}$ However, recent research suggests that objective measures may be necessary for accurate adherence measurement in complex disease such as CVD. ${ }^{44}$ According to a meta-analysis, correlations between adherence rates measured by self-report questionnaires and MEMS are much 
lower in CVD-related conditions like hypertension than in other diseases like HIV/AIDS; ${ }^{44}$ this may be due to the complex nature of CVD self-management. Yet, in routine clinical care, providers rarely have the time to accurately measure medication adherence; this suggests a need for enhanced measurement systems and a team-based approach to self-management.

One of the most popular forms of medication adherence telemonitoring is the use of a MEMS cap, a medication pill bottle with a microchip-equipped lid that passively measures medication adherence by date- and time-stamping electronically during each bottle-opening event. ${ }^{45}$ MEMS caps may be a useful tool in routine clinical care when used appropriately, although they are not without impediments like any other measurement system. However, limitations of MEMS caps are reactivity and technological failure. ${ }^{45}$ Consequently, researchers should use a 1- or 2-month run-in period to minimize measurement reactivity; corroborate MEMS data with another measure of adherence; report MEMS data continuously or use cut-offs determined by pharmacological properties and consequence of nonadherence to the medication; assess MEMS data using multilevel modeling; and properly train patients to use MEMS to improve their accuracy as an adherence measurement tool. ${ }^{45}$

Dichotomizing adherence is likely inappropriate in CVD patients. ${ }^{46}$ Researchers frequently dichotomize adherence based on a convention from the HIV/AIDS literature in which $\geq 80 \%$ is considered "adherent" because it is thought to provide optimal virologic suppression. ${ }^{30}$ The proper cut-off point should be determined for each medication formulation and the specific disease condition. ${ }^{30}$ But a recent study found that low-density lipoprotein cholesterol and blood pressure continue to reduce with adherence levels exceeding $80 \%$, suggesting that the optimal medication adherence level for cardiovascular conditions may exceed $80 \% .{ }^{47}$ Given $\mathrm{Wu}$ et al's finding that HF patients maximized event-free survival by adhering to their medications at an $88 \%$ rate, ${ }^{46}$ CVD patients may need to exceed $80 \%$ adherence to improve quality of life and outcomes.

Future studies that use an $80 \%$ cut-off point should also report continuous adherence values. ${ }^{30}$ This is an extremely important factor because many previously reported findings have been interpreted using an unsubstantiated practice (ie, false dichotomization), which has inappropriately shaped findings and clinical practice. For the sake of sound methodology and patients' lives, empirically derived cut-off points are the only points that a researcher should use in cases where adherence is not reported continuous.

\section{Comparing methods of medication adherence in CVD}

\section{Comparing self-report to MEMS caps}

Studies comparing CVD patients' self-reported and objectively measured medication adherence have demonstrated significant differences in adherence levels based on the strategies used to measure medication adherence. Previous studies have found a correlation between self-reported adherence and adherence measured by MEMS caps, though not in CVD. ${ }^{44}$ In a sample of 68 outpatients, most patients (67\%) overestimated their compliance when they used selfreported diaries, and an average of $30 \%$ of the self-reported diary entries were errantly recorded compared with the objective MEMS-produced data. ${ }^{48}$ Similarly, a study of hospitalized cardiac patients employed electronic monitoring and found adherence rates three times lower than in a study that utilized self-report, which suggests that CVD patients dramatically overestimate their self-reported adherence. ${ }^{49}$ In another comparison of self-report to objectively measured medication adherence, HF patients claimed to adhere to their medications $100 \%$ of the time; however, according to MEMS, only $76 \%$ of patients adhered to their medication at least $88 \%$ of the time. Thus, patients' estimated values do not necessarily predict medication-taking behavior, and individuals with CVD may have difficulty accurately predicting their true rate of medication adherence. ${ }^{50}$ This further corroborates the notion that patients with complex diseases need objective measurement tools to accurately assess the quality of their self-management, and they need care systems that target barriers to adherence through a comprehensive team approach.

\section{Present paper}

This paper reviews the literature on depression and objectively measured medication adherence in adult CVD patients. Based on the findings, the paper outlines a model that can serve as a solution to the current challenges.

\section{Methods}

The authors searched in PubMed and Google Scholar starting in April 2014 and limited search results to articles published in the past 20 years. They searched for studies that objectively measured medication adherence in CVD (eg, electronic monitoring, pill counts, and biological measures). The authors repeated their searches periodically (twice in 2015 and 2016 each) up until manuscript submission. The authors searched PubMed and Google Scholar using variants and combinations of the terms "depression," "depressive symptoms," 
"CVD," "medication adherence," and "objective." Initially, one author independently screened titles and abstracts to determine inclusion status; a second author verified the information after the first author completed the screening. If the second author found an article that met the team's inclusion criteria, then the first author searched that the reference list of that article for additional relevant studies. This process quickly reached a saturation point in which no new sources were found. In addition, the database search yielded thousands of blatantly irrelevant sources through Google Scholar. After sorting by relevance, the first author stopped screening articles when $>100$ consecutive articles were identified as being obviously irrelevant.

The authors also searched manually in the following key journals for additional relevant articles: JAMA, Circulation, Heart \& Lung, The Journal for Cardiovascular Nursing, European Heart Journal, and Patient Preference and Adherence. However, likely due to the number of articles already screened for inclusion, this process did not result in finding additional articles that were finally included in the team's results. Hence, numerous relevant articles were excluded (eg, focused solely on hypertension).

Included articles focused on medication adherence in CVD patients using objectively monitored medication adherence. For the purpose of this study, the team defined "medication adherence" as the amount of prescribed medication a patient took relative to the entire prescribed (or monitored) regimen.

The researchers most frequently excluded papers that used self-reported adherence, used animal models, or examined solely hypertension. The researchers may have missed some studies that met those criteria, particularly if the studies were not translated into English. Also, although other self-management behaviors are likely influenced by depression and affect outcomes, such as adherence to a low-salt diet, those behaviors far exceeded the scope of this paper, and thus studies focusing on other self-management behaviors were excluded.

Although previous reviews have looked at these topics separately, particularly using self-reported medication adherence, this paper aims to comprehensively summarize the literature using only the objective measures of medication adherence that seem to be most accurate in CVD patients. Given the limited research available using objective medication adherence techniques, the researchers created this review but were informed by a scoping ${ }^{51,52}$ rather than narrative framework. Furthermore, the search methodology was informed by PRISMA guidelines ${ }^{53}$ to enhance the quality of the review, but the available literature and scoping approach limited the researchers' ability to strictly adhere to those guidelines.

After screening and removing extraneous or unrelated studies, the researchers included three studies. Figure 1 shows

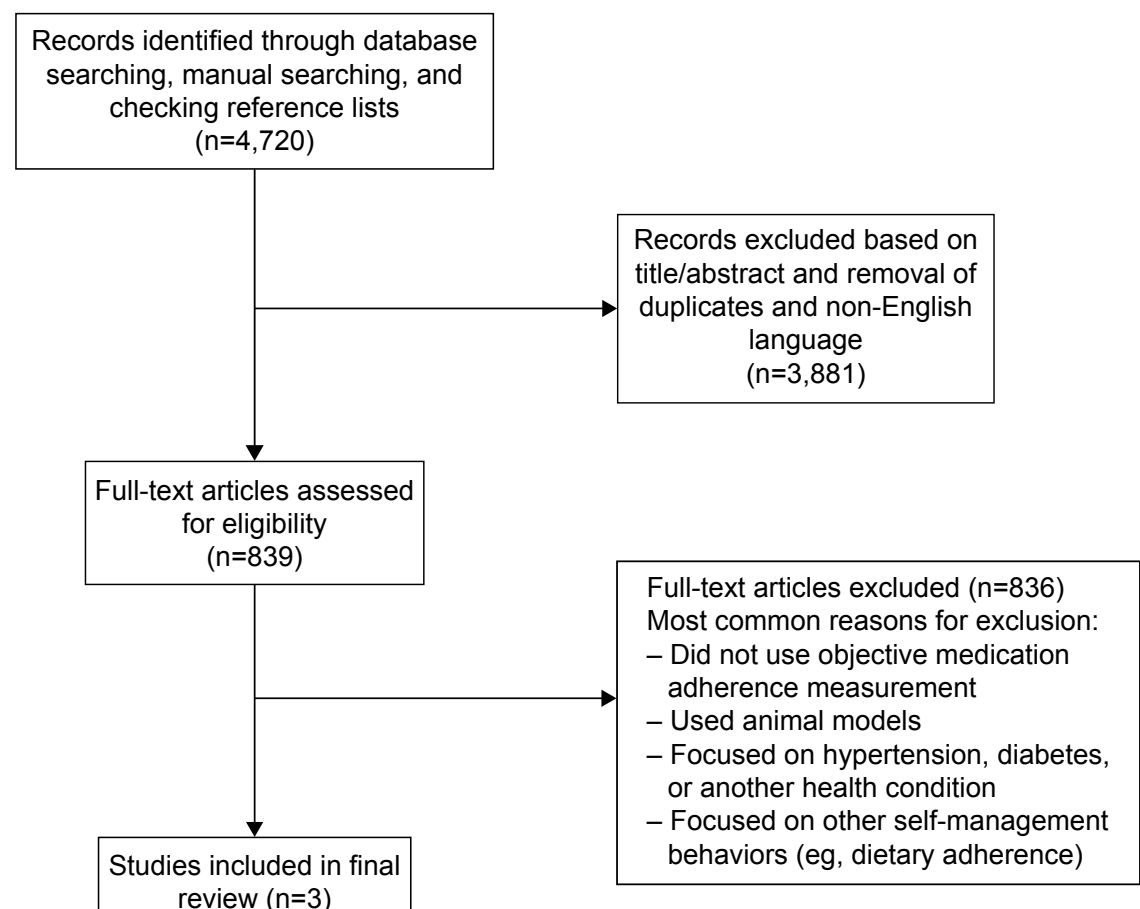

Figure I Flow diagram of study selection. 
the paper selection process. The researchers did not attempt to conduct a meta-analysis of the findings because of the small number of studies found and differences in methods of reporting medication adherence (ie, dichotomized reporting vs continuous values).

\section{Results}

\section{The relationship of depression and medication adherence in CVD}

Depression complicates CVD patients' ability to properly adhere to their medications and to comply with recommended health advice. For example, depression predicts failure to complete a 12 -week phase II cardiac rehabilitation program. ${ }^{54}$ Persistent depressive symptoms are associated with poor adherence to health behaviors ${ }^{4}$ and high risk for mortality. ${ }^{27,55}$

A landmark study by Carney et al in 1995 examined the relationship between major depression and medication adherence in 55 older adults with CVD. The study involved a depression diagnosis assigned by diagnostic interview and objective adherence to $81 \mathrm{mg}$ aspirin measured by an unobtrusive electronic monitor. ${ }^{56}$ Nondepressed patients adhered to their medications $69 \%$ of the days when they were monitored, but depressed patients adhered to their medication only $45 \%$ of the days monitored. The study showed clinically significant differences between depressed and nondepressed CVD patients' adherence to a vitally important cardiac medication. ${ }^{56}$ Depression clearly harms self-management in CVD.

Another study that assessed the relationship between depression and medication adherence tested whether reductions in depressive symptoms preceded improved adherence to aspirin over 3 months in 172 acute coronary syndrome patients. ${ }^{49}$ In that study, researchers measured aspirin adherence using MEMS, and they assessed depression with the Beck Depression Inventory (BDI) during hospitalization and 1 and 3 months after hospitalization. Using MEMS, adequate adherence was defined as taking aspirin as prescribed at least $80 \%$ of the monitored days. Depression severity was associated with nonadherence in a gradient manner: $15 \%$ of nondepressed, $29 \%$ of mildly depressed, and $37 \%$ of moderately to severely depressed patients were nonadherent. The study determined that severely depressed patients were 3.7 times more likely to be nonadherent than nondepressed patients after controlling for potential confounders. Furthermore, adherence increased in patients whose depressive symptoms improved, and it decreased in patients whose depressive symptoms worsened. ${ }^{49}$
According to follow-up analyses, the mean percentage of days when patients properly adhered to their aspirin regimen was lower among persistently depressed patients than in remittent depressed and persistently nondepressed patients. ${ }^{57}$ These results remained after controlling for medical comorbidities and baseline depressive symptom severity. Patients whose BDI scores remitted below 10 after 3 months posthospitalization adhered more than persistently depressed patients, and they ultimately became equally as adherent as persistently nondepressed patients. ${ }^{57}$ However, improvements in depression did not show perfect adherence rates, suggesting that other factors influence this relationship.

The relationship between depression and medication adherence in CVD is complicated and seems to be moderated by additional factors. Treating depression alone is unlikely to completely improve medication adherence, and onedimensional approaches have been largely unsuccessful at modifying this relationship. As the integrated care approach gains popularity and dissemination, the scientific community will likely better understand that the integrated care treatment model is ideally constructed to address this major clinical problem. Future research should determine whether the integrated care model improves health and reduces costs for patients and health care systems; significant progress in CVD management could result from a shift in health care delivery models.

\section{Discussion}

In the United States, currently, CVD is the leading cause of death of American adults, ${ }^{1}$ and many CVD patients experience depressive symptoms. However, attempts to reduce depressive symptoms through pharmacotherapy and psychotherapy have not considerably reduced mortality rates. This is likely because depressive symptoms tend to impede ideal adherence behavior, which contributes to poorer outcomes, including mortality. Nevertheless, simply targeting medication adherence behavior may not resolve this association and completely improve outcomes. Instead, moderating factors (eg, the medical regimen's complexity) likely influence the relationship between depression and medication adherence in CVD.

Managing the CVD patient is a responsibility that extends beyond a single provider. Instead, treatment providers should adopt a team-based, integrated approach for addressing associated medical, psychosocial, and cultural processes.

\section{Integrated care: a possible solution}

At present, $20 \%$ of primary care office visits are mental health-related, ${ }^{58}$ and depression goes undetected in $>50 \%$ of 
primary care patients..$^{59}$ Providers rarely address adherence during outpatient health care appointments, and practitioners sometimes assume that the patients are taking their medications as prescribed.

In an integrated care health care model, primary care and behavioral health clinicians work synchronously with patients and families in a systematic, cost-effective manner. Their shared objective is to provide comprehensive patientcentered care and to address both the patient's physical health and behavioral health needs. ${ }^{60}$

\section{The Affordable Care Act}

The Patient Protection and Affordable Care Act (hereafter referred to as the Affordable Care Act) encourages providers to integrate physical and behavioral health services, which helps them to deliver care more coherently and to reduce excess costs. This benefits both patients and health care providers. For example, as many individuals struggle to accurately describe their health history to different practitioners, integrated care can reduce issues in physical and mental health treatment stemming from limited cross-coordination. The Affordable Care Act also allows for increased coverage of home-based health care. This represents an extension of the patient-centered medical home, a model of delivery focused on patient-centered, coordinated health care through a team of health care providers led by the patient's primary care physician.

\section{Integrated care teams}

Patient-centered, integrated care teams have already shown significant promise. The Veteran's Health Administration (VHA) has implemented an integrated care model throughout its system with great success. Changes in service delivery resulting from the VHA's integrated health care model have led to improved recognition of mental health issues in primary care patients, delivery of evidence-based care, and patient engagement in mental health services. ${ }^{61}$ In addition, among high-risk, complex patients, the patient-centered medical home system seems to be associated with improved chronic disease management and outcomes. ${ }^{62}$ Furthermore, establishing a health behavior coordinator position at each Veterans Affairs hospital has helped to implement integrated care in veteran's services by providing training to primary care physicians, physicians' assistants, nurses, and other health care providers. Additional integration of physical and behavioral health services may increase the delivery of evidence-based mental health treatment and could possibly begin to address mental health issues that often interfere with adherence to medical interventions in primary care settings. Although this system is working well in the VHA, and despite its promise for complex, sick patients, the broader health care community has been slow to adopt a truly integrated model.

The typical integrated care team's structure is a collaborative system in which each member contributes his or her expertise to shape patient care. Each provider on the team can contribute to improving treatment adherence and self-management among CVD patients. Table 1 provides examples of unique contributions that each team member can make to provide depression and poor medication adherence in CVD patients. Although physicians could refer patients with depression to additional services that address both the depressive symptoms and their effects on self-management, these patients' needs would be best met by an assembly of specialty service providers who work together toward numerous treatment targets.

Furthermore, the most likely course of CVD involves disease progression that over time requires more care and intervention to maximize the chance of survival; creating these treatment teams early on may improve a patient's outcomes. That is, it could improve the quality of life, prolong longevity, and decrease health care expenditures by preventing unnecessary rehospitalization due to poor self-management and delaying need for surgical intervention. Ideally, treatment teams would be composed of as many of the following as possible: a primary physician (primary care or specialty), a physician's assistant, one or more advanced practice and registered nurses, a psychologist, a psychiatrist, a social worker, a dietician, one or more physical and occupational therapists, and a pharmacist. As represented in Table 1, each member would function in a unique role to improve the patient's overall health and well-being, and each member could contribute meaningful care to reduce depression and improve self-management. Furthermore, some responsibilities could be shared among group members, as described in Table 2. In addition, as the integrative team continues to collaborate, it may become necessary to clarify roles over time and update other less-involved team members (eg, outside consultants) with changes to the treatment plan or clinical status. Medical students and residents should be appropriately trained in the integrated care model during the course of their medical training, while established practitioners should be provided with appropriate continuing education opportunities. As is the case with any framework shift, some practitioners may be resistant to changing protocols and may require additional information about how integrated care can benefit both providers and patients, or they may require additional incentives (eg, financial incentives). 
Table I Role of integrated care team members in targeting depression and medication adherence in CVD patients

\begin{tabular}{|c|c|}
\hline Type of professional & $\begin{array}{l}\text { Role in integrated care team members in targeting depression and medication adherence in CVD } \\
\text { patients }\end{array}$ \\
\hline $\begin{array}{l}\text { Primary medical doctor } \\
\text { and physician's assistant }\end{array}$ & $\begin{array}{l}\text { - Monitor cardiac status } \\
\text { - Diagnose medical conditions } \\
\text { - Prescribe medications for mental health, CVD, and other health conditions } \\
\text { - Monitor symptoms and side effects of medications } \\
\text { - Perform or refer patients for procedures necessary for disease management } \\
\text { - Educate patients about strategies for preventing future disease and disease progression } \\
\text { - Modify patients' overall treatment plan based on a stepped care approach } \\
\text { - Refer to consultation with specialists as necessary, particularly to psychiatry if depression does not respond to } \\
\text { depression management protocol }\end{array}$ \\
\hline $\begin{array}{l}\text { Advanced practice and } \\
\text { registered nurse }\end{array}$ & $\begin{array}{l}\text { - Monitor cardiac status } \\
\text { - Monitor other medical co-morbidities } \\
\text { - Medication management through pre-filling pill boxes, clarifying medication instructions, and collaborating with } \\
\text { - Monitor for precipitants of rehospitalization, including depression } \\
\text { - Facilitate mail-order refills or pharmacy reminders and other tools to ensure that patient does not run out of } \\
\text { medication }\end{array}$ \\
\hline Psychologist & $\begin{array}{l}\text { - Treat mental health, particularly depression and health-interfering behaviors such as excessive rest periods } \\
\text { following pain flare-ups or emotional eating } \\
\text { - Increase medication adherence through motivational interviewing, interventions to manage cognitive } \\
\text { impairment, CBT, and ACT } \\
\text { - Monitor and test for cognitive factors that may be limiting self-management; refer for neuropsychological } \\
\text { testing as necessary } \\
\text { - Clarify gaps in health literacy or patient understanding of their conditions, medications, and treatment options } \\
\text { - Engage family in patient's treatment by providing recommendations for how the family can contribute to their } \\
\text { loved one's health }\end{array}$ \\
\hline Psychiatrist & $\begin{array}{l}\text { - Evaluate patient for medication if referred from primary doctor or physician's assistant } \\
\text { - Consider ways to simplify the regimen and finally improve medication adherence } \\
\text { - Engage families in patients' treatment by providing recommendations on how they can support their loved } \\
\text { one's mental health } \\
\text { - Provide psychoeducation about proper ways to take medication } \\
\text { - Evaluate for need of higher level of care (eg, inpatient psychiatric hospitalization) and facilitate with admitting } \\
\text { attending if necessary }\end{array}$ \\
\hline Social worker & $\begin{array}{l}\text { - Case management } \\
\text { - Screen for poor mental health, poor self-management, poor diet, cognition, and sedentary lifestyle } \\
\text { - Provide supportive counseling as necessary } \\
\text { - Measure mental and physical health frequently to shape the course of treatment } \\
\text { - Secure patient access to community resources that can improve patient's longevity and quality of life } \\
\text { - Manage financial burden of disease management with patient }\end{array}$ \\
\hline Dietician & $\begin{array}{l}\text { - Provide psychoeducation about proper CVD diet } \\
\text { - Monitor for dietary intake that is contraindicated with prescribed medications and supplements } \\
\text { - Collaborate with patient and family to meet patient's dietary goals over time }\end{array}$ \\
\hline $\begin{array}{l}\text { Physical and } \\
\text { occupational therapist }\end{array}$ & $\begin{array}{l}\text { - Prescribe physical activity plan that reflects patient's willingness, preference, and ability to be physically active } \\
\text { - Monitor for sedentary behavior and use brief targeting interventions with goal-setting to help patient achieve } \\
\text { physical activity goals } \\
\text { - Monitor for behavioral inactivation or physical agitation (particularly as a symptom of mental health } \\
\text { disturbance) } \\
\text { - Assess for motor problems that may negatively influence self-management (eg, poor fine motor skills that make } \\
\text { getting pill bottles open difficult) } \\
\text { - If possible, treat for motor difficulties, chronic pain, etc to restore functioning }\end{array}$ \\
\hline Pharmacist & $\begin{array}{l}\text { - Monitor for drug reactions and lab levels that suggest a medication adjustment may be necessary, particularly } \\
\text { following medication changes and rehospitalization } \\
\text { - Collaborate with other prescribing providers to simplify the regimen as much as possible } \\
\text { - Monitor for mental health symptoms that may be a consequence of another medication } \\
\text { - Revise medication regimen to minimize adverse effects and optimize efficacy } \\
\text { - Ensure patient and family are aware of medications, dosages, and potential side effects }\end{array}$ \\
\hline
\end{tabular}

Abbreviations: ACT, acceptance and commitment therapy; CVD, cardiovascular disease; CBT, cognitive behavioral therapy. 
Table 2 Shared responsibilities of integrated care team members in targeting CVD patients' depression and medication adherence

\begin{tabular}{ll}
\hline Patient care & - Monitor for overall safety (eg, fall risk, suicidal ideation, and medication interactions) \\
- Maintain an up-to-date treatment plan, electronic patient health record, and resource list for patient and family \\
- Maintain patient's health, family, and community as the guiding point of all care decisions \\
Collaboration with & - Connect patient to acute care when needed and facilitate case coordination with emergency or hospital providers \\
treatment team & - Encourage increased patient accessibility to care through an electronic health record, a patient portal, after-hours or \\
& - Meekend visits, and home visits when safe and appropriate \\
Collaboration with & - Introduce and revisit advance directives and health care goals with the patient and family \\
patient and family & - Collaborate with the patient and family to reduce hospitalization and improve quality of life
\end{tabular}

Abbreviation: CVD, cardiovascular disease.

Furthermore, telemedicine could unite practitioners to form teams across rural or underserved areas; future policy development is necessary to allow for comparable reimbursement of these services. Integrated teams with adequate resources should ideally conduct outcomes and cost-effectiveness research so that these data can be used to justify policy shifts and a change from a fee-for-service model to one that supports interdisciplinary chronic disease management.

\section{Feasibility}

Given the ways in which health care systems are changing in the United States and worldwide based on policy changes, electronic health records, and other care innovations, this approach will likely become more feasible over time. For example, the Affordable Care Act is facilitating a movement to expand integrated care services in community primary care settings. Several studies have already demonstrated the feasibility of integrated care in HF, ${ }^{63}$ diabetes ${ }^{64}$ and stroke.${ }^{65}$ These studies indicated that an integrated care team produced up to a $70 \%$ increase of patients taking a more active role in their health care, ${ }^{64}$ improved patient outcomes (eg, blood pressure, cholesterol, and quality of life) ${ }^{64,65}$ and improved satisfaction from staff and patients, ${ }^{64}$ contributing to lower admissions rates by $20 \%$ and lower health care costs. ${ }^{63}$ In addition, the VHA already has a model for patient-aligned care teams and health behavior coordinator positions to increase patient-provider partnership in care and provide health behavior change training to clinical staff. Such programs have been successful thus far and serve as models for other health care providers.

Integrated care benefits not only providers (through better communication, medical management, health education, and case coordination) but also patients (through increased convenience, decreased stigma, higher quality care, and quicker appointments) ${ }^{66}$ Given the changing health care system, the population's increasing longevity and medical complexity, and the demands of coaching patients to achieve adequate self-management, the research community should further explore integrated care systems and evidence-based practice as exemplary opportunities for reducing depression, increasing medication adherence and other components of self-management, and leading the field toward improved quality of life and outcomes in individuals with any chronic illness.

\section{Potential interventions}

Numerous available interventions target medication adherence behavior. A multidimensional, tailored approach that targets health literacy, encourages self-monitoring, utilizes automatic alerts when prescriptions are not filled on time, and employs blister packs and pill boxes may improve adherence in adults with CVD and other chronic illnesses. ${ }^{28,67}$ However, discrepancies exist in the literature. Some studies report that electronic objective monitoring burdens and challenges individuals with CVD. ${ }^{68}$ However, although it may seem counterintuitive that CVD patients, and particularly older patients, can handle electronic monitoring, feasibility studies suggest they can. ${ }^{69}$

Health care professionals may also find that interventions using phone counseling or nurses as case managers in primary care may also be effective and feasible, as the interventions are short and do not require patients to leave their homes. ${ }^{70}$ Studies have associated such interventions with improved depression for individuals. ${ }^{70,71}$ Other treatments include antidepressants, CBT, physical activity, and cardiac rehabilitation programs. ${ }^{72}$ Motivational counseling may be another effective method for improving adherence in CVD patients. ${ }^{73,74}$ Antidepressants are also available, but it is important to note that SSRIs are preferable to tricyclic antidepressants because of the latter's significant cardiovascular side effects. ${ }^{75}$ Further randomized clinical trials are needed to determine whether SSRIs, psychotherapy, or both can reduce cardiac events and mortality in CVD. ${ }^{76}$ 
In addition, interventions can target different populations to maximize success. Interventions that target nonadhering patients may have a larger effect on medication adherence ${ }^{77}$ and be more cost-effective than other studies. Programs that target both the patient and the caregiver, especially significant others, in improving health behavior of CVD patients ${ }^{78}$ may produce sustainable improved outcomes.

\section{Overall limitations}

A major methodological issue limits the medication adherence research literature: many well-designed studies rely on self-reported adherence behavior. ${ }^{79}$ As evidenced in the present research, self-reported estimates of medication adherence may overestimate medication adherence behavior in complicated chronic illnesses such as CVD. ${ }^{44}$ Future research should measure adherence objectively (eg, through pill counts or electronic monitoring). However, electronic devices also suffer from limitations such as "fake" medication-taking behavior to silence an alarm or a patient misplacing the medication after the device has already recorded a medication-taking event. Future technological designs should attempt to minimize discrepancies between a technologically documented event and real-time medicationtaking behavior.

As previously stated, the authors of this study were unable to include papers not published in English, and future reviews should examine research conducted in non-English-speaking countries, if possible, to better understand the worldwide literature to date.

\section{Future research}

The health care research community has advanced exceptionally to understand the relationship between depression and medication adherence, and future research will dramatically affect clinical settings. Clinicians need easy, systematic, and noninvasive screenings for depression and medication adherence to use in outpatient cardiology routine visits. ${ }^{80}$ Quick-targeted interventions using stepped care are also needed and will likely incorporate large, well-powered trials of antidepressants and psychotherapies. ${ }^{81}$

In addition, many current medication adherence measurement strategies are expensive (eg, MEMS), ${ }^{33}$ and accessible interventions will need to be cost-effective and widely available. As the pathophysiological link between depression and CVD remains unclear, there is a similar lack in pharmacotherapy that targets the dysregulated physiology that might help explain the increased morbidity and mortality in patients with both CVD and depression. ${ }^{82}$ Therefore, future pharmaceutical and behavioral research can target this link as a point for intervention.

\section{Overcoming methodological issues}

Future research must address methodological issues. The field should develop a consistent method for reporting medication adherence, as comparing adherence rates across studies and conditions remains difficult. ${ }^{30}$ Moreover, the present review only incorporated high-quality publications using objective measurements; readers should use caution to evaluate the quality of the evidence and the methodology and inclusion criteria while reading other reviews in this area. Furthermore, interventions continue to focus on a single disease, but a typical patients takes multiple medications for various conditions. ${ }^{28}$ Future interventions should provide a menu-driven, patient-centered approach to maximize the extent to which research translates to real-world nonadherence problems; menu-driven, patient-centered approaches can easily be tailored to the individual and address a wide variety of self-management challenges. ${ }^{28}$ In addition, prospective studies are needed to understand directionality and determine whether poor medication adherence causes individuals to become depressed. Prospective studies must determine the specifics of nonadherence behavior, which may include failure to begin, execute, or continue the regimen. In light of great advances of medicines in the past decade, future research should target methods in which health care providers can encourage patients to take their medications as prescribed.

\section{Directions for future research in integrated care}

Much work in integrated care is still needed. Further studies on the feasibility of integrated care teams would help identify the team's place in the health care structure and its impact on the overall health care costs. In addition, existing integrated teams could provide the field with valuable data on patient outcomes and cost-effectiveness to better support interdisciplinary chronic disease management. As integrated care transitions from idea to practice, continued research is needed to understand the impact of a team-based approach on depression, quality of life, and outcomes. Future research should identify the most salient team components that are responsible for long-term improvements in self-management. Further, as technology continues to revolutionize health care practice, future research should examine the barriers and opportunities for technology's role as a tool in improving care. 
Ongoing dissemination and implementation of integrated care systems should embody an iterative process in which all key stakeholders are involved, including patients and their families. Programs should be realistic, capitalize on the team's strengths, and be aspirational in nature. Finally, even with the integrated care model's increasing popularity, many patients remain underserved. Therefore, providers and governments should target health disparities to reduce health-related inequities across communities and patient populations.

Although depression and poor medication adherence are difficult to detect and repair, both are modifiable risk factors for decreased event-free survival in CVD. However, simply treating depression does not perfect medication adherence, just as targeting poor medication adherence does not resolve depression. Many of the moderating factors that influence the relationship are either modifiable or easy to detect. Although the depression-adherence relationship is complex and poorly understood currently, future research may illuminate the etiology of the relationship and the proper intervention that will improve CVD patients' quality of life and outcomes.

\section{Acknowledgments}

The authors wish to thank Drs Yossef Ben-Porath, Dale Bond, John Gunstad, Joel Hughes, Karen Oliver, Pamela SteadmanWood, and J Graham Thomas for their advice during this project's formative stages. They also thank Ryan J Voorhees for his editorial consultation.

\section{Disclosure}

The authors report no conflicts of interest in this work.

\section{References}

1. American Heart Association. Heart disease and stroke statistics - 2013 update: a report from the American Heart Association. Circulation. 2013;127(1):e6-e245.

2. Frasure-Smith N, Lespérance F. Recent evidence linking coronary heart disease and depression. Can J Psychiatry. 2006;51(12):730-737.

3. Barth J, Schumacher M, Herrmann-Lingen C. Depression as a risk factor for mortality in patients with coronary heart disease: a meta-analysis. Psychosom Med. 2004;66(6):802-813.

4. Kronish IM, Rieckmann N, Halm EA, et al. Persistent depression affects adherence to secondary prevention behaviors after acute coronary syndromes. J Gen Intern Med. 2006;21(11):1178-1183.

5. Carney RM, Freedland KE. Depression in patients with coronary heart disease. Am J Med. 2008;121(11B):S20-S27.

6. Thombs BD, Bass EB, Ford DE, et al. Prevalence of depression in survivors of acute myocardial infarction. J Gen Intern Med. 2006; 21(1):30-38.

7. Rutledge T, Reis VA, Linke SE, Greenberg BH, Mills PJ. Depression in heart failure: a meta-analytic review of prevalence, intervention effects, and associations with clinical outcomes. J Am Coll Cardiol. 2006;48(8): 1527-1537.

8. Powell LH, Catellier D, Freedland KE, et al. Depression and heart failure in patients with a new myocardial infarction. Am Heart J. 2005; 149(5):851-855.
9. Hayes SN. Broken-hearted women: the complex relationship between depression and cardiovascular disease. Womens Health (Lond). 2009;5(6):709-725.

10. Spijkerman TA, van den Brink RH, Jansen JH, Crijns HJ, Ormel J. Who is at risk of post-MI depressive symptoms? J Psychosom Res. 2005;58(5):425-432.

11. Johnson TJ, Basu S, Pisani BA, et al. Depression predicts repeated heart failure hospitalizations. J Card Fail. 2012;18(3):246-252.

12. Moser DK, Doering LV, Chung ML. Vulnerabilities of patients recovering from an exacerbation of chronic heart failure. Am Heart J. 2005;150(5):984.e7-984.e13.

13. Reese RL, Freedland KE, Steinmeyer BC, Rich MW, Rackley JW, Carney RM. Depression and rehospitalization following acute myocardial infarction. Circulation. 2011;4(6):626-633.

14. van Melle JP, de Jonge P, Spijkerman TA, et al. Prognostic association of depression following myocardial infarction with mortality and cardiovascular events: a meta-analysis. Psychosom Med. 2004;66(6): 814-822.

15. Powell LH, Calvin JE Jr, Richardson D, et al. Self-management counseling in patients with heart failure: primary results from the Heart Failure Adherence and Retention Trial (HART). JAMA. 2010; 304(12):1331-1338.

16. Freedland KE, Carney RM, Rich MW. Impact of depression on prognosis in heart failure. Heart Fail Clin. 2011;7(1):11-21.

17. Testa G, Cacciatore F, Galizia G, et al. Depressive symptoms predict mortality in elderly subjects with chronic heart failure. Eur J Clin Invest. 2011;41(12):1310-1317.

18. Carney RM, Blumenthal JA, Catellier D, et al. Depression as a risk factor for mortality after acute myocardial infarction. Am J Cardiol. 2003;92(11):1277-1281.

19. Jiang W, Alexander J, Christopher E, et al. Relationship of depression to increased risk of mortality and rehospitalization in patients with congestive heart failure. Arch Intern Med. 2001;161(15):1849-1856.

20. Sherwood A, Blumenthal JA, Hinderliter AL, et al. Worsening depressive symptoms are associated with adverse clinical outcomes in patients with heart failure. J Am Coll Cardiol. 2011;57(4):418-423.

21. Joynt KE, Whellan DJ, O'Connor CM. Why is depression bad for the failing heart? A review of the mechanistic relationship between depression and heart failure. J Card Fail. 2004;10(3):258-271.

22. Carney RM, Freedland KE, Steinmeyer B, et al. History of depression and survival after acute myocardial infarction. Psychosom Med. 2009; 71(3):253-259.

23. Berkman LF, Blumenthal J, Burg M, et al. Effects of treating depression and low perceived social support on clinical events after myocardial infarction: The Enhancing Recovery in Coronary Heart Disease Patients (ENRICHD) Randomized Trial. JAMA. 2003;289(23):3106-3116.

24. Carney RM, Freedland KE, Steinmeyer B, et al. Depression and five year survival following acute myocardial infarction: a prospective study. J Affect Disord. 2008;109:133-138.

25. Chung ML, Dekker RL, Lennie TA, Moser DK. Antidepressants do not improve event-free survival in patients with heart failure when depressive symptoms remain. Heart Lung. 2013;42(2):85-91.

26. Glassman AH, Bigger JT Jr, Gaffney M. Psychiatric characteristics associated with long-term mortality among 361 patients having an acute coronary syndrome and major depression: seven-year follow-up of SADHART participants. Arch Gen Psychiatry. 2009;66(9):1022-1029.

27. Scherrer JF, Chrusciel T, Garfield LD, et al. Treatment-resistant and insufficiently treated depression and all-cause mortality following myocardial infarction. Br J Psychiatry. 2012;200(2):137-142.

28. Bosworth H, Granger BB, Mendys P, et al. Medication adherence: a call for action. Am Heart J. 2011;162(3):412-424.

29. Gilberg K, Laouri M, Wade S, Isonaka S. Analysis of medication use patterns: apparent overuse of antibiotics and underuse of prescription drugs for asthma, depression, and CHF. J Manag Care Pharm. 2003;9(3):232-237.

30. Ho PM, Bryson CL, Rumsfeld JS. Medication adherence its importance in cardiovascular outcomes. Circulation. 2009;119(23):3028-3035. 
31. Wu JR, Moser DK, Lennie TA, Burkhart PV. Medication adherence in patients who have heart failure: a review of the literature. Nursing Clinics of North America. 2008;43(1):133-153.

32. Aggarwal B, Mosca L. Lifestyle and psychosocial risk factors predict non-adherence to medication. Ann Behav Med. 2010;40(2):228-233.

33. World Health Organization. Adherence to long-term therapies: evidence for action; 2003. Available from: http://www.who.int/chp/knowledge/ publications/adherence_report/en/. Accessed November 10, 2016.

34. Ansell BJ. Not getting to goal: the clinical costs of noncompliance. J Manag Care Pharm. 2008;14(6 Suppl B):9-15.

35. Riegel B, Lee C, Ratcliffe S, et al. Predictors of objectively measured medication nonadherence in adults with heart failure. Circ Heart Fail. 2012;5(4):430-436.

36. Calvin JE, Shanbhag S, Avery E, Kane J, Richardson D, Powell L. Adherence to evidence based guidelines for heart failure in physicians and their patients: lessons from the Heart Failure Adherence Retention Trial (HART). Congest Heart Fail. 2012;18(2):73-78.

37. Brown TM, Siu K, Walker D, Pladevall-Vila M, Sander S, Mordin M. Development of a conceptual model of adherence to oral anticoagulants to reduce risk of stroke in patients with atrial fibrillation. J Manag Care Pharm. 2012;18(5):351-362.

38. Annema C, Luttik ML, Jaarsma T. Reasons for readmission in heart failure: perspectives of patients, caregivers, cardiologists, and heart failure nurses. Heart Lung. 2009;38(5):427-434.

39. Wu JR, Moser DK, Chung ML, Lennie TA. Objectively measured, but not self-reported, medication adherence independently predicts event-free survival in patients with heart failure. J Card Fail. 2008;14(3):203-210.

40. Wu JR, Moser DK, Chung ML, Lennie TA. Predictors of medication adherence using a multidimensional adherence model in patients with heart failure. J Card Fail. 2008;14(7):603-614.

41. Osterberg L, Blaschke T. Adherence to medication. $N$ Engl J Med. 2005;353(5):487-497.

42. Gehi A, Haas D, Pipkin S, Whooley MA. Depression and medication adherence in outpatients with coronary heart disease: findings from the Heart and Soul Study. Arch Intern Med. 2005;165(21):2508-2513.

43. Stirratt MJ, Dunbar-Jacob J, Crane HM, et al. Self-report measures of medication adherence behavior: recommendations on optimal use. Transl Behav Med. 2015;5(4):470-482.

44. Shi L, Liu J, Koleva Y, Fonseca V, Kalsekar A, Pawaskar M. Concordance of adherence measurement using self-reported adherence questionnaires and medication monitoring devices. Pharmacoeconomics. 2010; 28(12):1097-1107.

45. Cook P, Schmiege S, McClean M, Aagaard L, Kahook M. Practical and analytic issues in the electronic assessment of adherence. West J Nurs Res. 2012;34(5):598-620.

46. Wu JR, Moser DK, de Jong MJ, et al. Defining an evidence-based cutpoint for medication adherence in heart failure. Am Heart J. 2009; 157(2):285-291

47. Bryson CL, Au DH, Young B, McDonell MB, Fihn SD. A refill adherence algorithm for multiple short intervals to estimate refill compliance (ReComp). Med Care. 2007;45(6):497-504.

48. Straka RJ, Fish JT, Benson SR, Suh JT. Magnitude and nature of noncompliance with treatment using isosorbide dinitrate in patients with ischemic heart disease. J Clin Pharmacol. 1996;36(7):587-594.

49. Rieckmann N, Gerin W, Kronish IM, et al. Course of depressive symptoms and medication adherence after acute coronary syndromes. $\mathrm{J} \mathrm{Am}$ Coll Cardiol. 2006;48(11):2218-2222.

50. Nieuwenhuis MM, Jaarsma T, van Veldhuisen DJ, van der Wal MH. Self-reported versus 'true' adherence in heart failure patients: a study using the Medication Event Monitoring System. Neth Heart J. 2012; 20(7-8):313-319.

51. Arksey H, O’Malley L. Scoping studies: towards a methodological framework. Int J Soc Res Methodol. 2005;8(1):19-32.

52. Levac D, Colquhoun H, O'Brien KK. Scoping studies: advancing the methodology. Implement Sci. 2010;5(1):1-9.

53. Moher D, Liberati A, Tetzlaff J, Altman DG. Preferred reporting items for systematic reviews and meta-analyses: the PRISMA statement. Ann Intern Med. 2009;151(4):264-269.
54. Casey E, Hughes JW, Waechter D, Josephson R, Rosneck J. Depression predicts failure to complete phase-II cardiac rehabilitation. J Behav Med. 2008;31:421-431.

55. Carney RM, Blumenthal JA, Freedland KE, et al. Depression and late mortality after myocardial infarction in the Enhancing Recovery in Coronary Heart Disease (ENRICHD) study. Psychosom Med. 2004;66(4):466-474.

56. Carney RM, Freeland KE, Eisen SA, Rich MW, Jaffe AS. Major depression and medication adherence in elderly patients with coronary artery disease. Health Psychol. 1995;14(1):88-90.

57. Rieckmann N, Kronish IM, Haas D, et al. Persistent depressive symptoms lower aspirin adherence after acute coronary syndromes. Am Heart J. 2006;152(5):922-927.

58. Center for Disease Control and Prevention. Percentage of mental healthrelated primary care office visits, by age group - National Ambulatory Medical Care Survey, United States, 2010. Morbid Mortal Wkly Rep. 2014;63(47):1118.

59. Mitchell AJ, Vaze A, Rao S. Clinical diagnosis of depression in primary care: a meta-analysis. Lancet. 2009;374(9690):609-619.

60. Green LA, Cifuentes M. Advancing care together by integrating primary care and behavioral health. J Am Board Fam Med. 2015; 28(Suppl 1):S1-S6.

61. Pomerantz AS, Kearney LK, Wray LO, Post EP, McCarthy JF. Mental health services in the medical home in the Department of Veterans Affairs: factors for successful integration. Psychol Serv. 2014;11(3):243-253.

62. O'Toole TP, Pirraglia PA, Dosa D, et al. Building care systems to improve access for high-risk and vulnerable veteran populations. J Gen Intern Med. 2011;26(2):683-688.

63. Comin-Colet J, Verdu-Rotellar JM, Vela E, et al. Efficacy of an integrated hospital-primary care program for heart failure: a populationbased analysis of 56,742 patients. Rev Esp Cardiol (Engl Ed). 2014; 67(4):283-293.

64. Ciccone MM, Aquilino A, Cortese F, et al. Feasibility and effectiveness of a disease and care management model in the primary health care system for patients with heart failure and diabetes (project Leonardo). Vasc Health Risk Manag. 2010;6:297-305.

65. Kamm CP, Schmid JP, Muri RM, Mattle HP, Eser P, Saner H. Interdisciplinary cardiovascular and neurologic outpatient rehabilitation in patients surviving transient ischemic attack or stroke with minor or no residual deficits. Arch Phys Med Rehabil. 2014;95(4):656-662.

66. Gallo JJ, Zubritsky C, Maxwell J, et al. Primary care clinicians evaluate integrated and referral models of behavioral health care for older adults: results from a multisite effectiveness trial (PRISM-e). Ann Fam Med. 2004;2(4):305-309.

67. Murray MD, Morrow DG, Weiner M, et al. A conceptual framework to study medication adherence in older adults. Am J Geriatr Pharmacother. 2004;2(1):36-43.

68. Bauer LK, Caro MA, Beach SR, et al. Effects of depression and anxiety improvement on adherence to medication and health behaviors in recently hospitalized cardiac patients. Am J Cardiol. 2012;109(9):1266-1271.

69. Haberer JE, Robbins GK, Ybarra M, et al. Real-time electronic adherence monitoring is feasible, comparable to unannounced pill counts, and acceptable. AIDS Behav. 2012;16(2):375-382.

70. McLaughlin TJ, Aupont O, Bambauer KZ, et al. Improving psychologic adjustment to chronic illness in cardiac patients. J Gen Intern Med. 2005;20(12):1084-1090.

71. Morgan MA, Coates MJ, Dunbar JA, Reddy P, Schlicht K, Fuller J. The TrueBlue model of collaborative care using practice nurses as case managers for depression alongside diabetes or heart disease: a randomised trial. BMJ Open. 2013;3(1):1-11.

72. Lichtman JH, Bigger JT Jr, Blumenthal JA, et al. Depression and coronary heart disease recommendations for screening, referral, and treatment: a science advisory from the American Heart Association Prevention Committee of the Council on Cardiovascular Nursing, Council on Clinical Cardiology, Council on Epidemiology and Prevention, and Interdisciplinary Council on Quality of Care and Outcomes Research: Endorsed by the American Psychiatric Association. Circulation. 2008;118(17): $1768-1775$. 
73. Bouvy M, Heerdink E, Urquhart J, Grobbee D, Hoes A, Leufkens H. Effect of a pharmacist-led intervention on diuretic compliance in heart failure patients: a randomized controlled study. J Card Fail. 2003;9(5):404-411.

74. van Dalem J, Krass I, Aslani P. Interventions promoting adherence to cardiovascular medicines. Int J Clin Pharm. 2012;34(2):295-311.

75. Zellweger MJ, Osterwalder RH, Langewitz W, Pfisterer ME. Coronary artery disease and depression. Eur Heart J. 2004;25(1):3-9.

76. Davidson KW, Kupfer DJ, Bigger JT, et al. Assessment and treatment of depression in patients with cardiovascular disease: National Heart, Lung, and Blood Institute Working Group Report. Psychosom Med. 2006;68(5):645-650.

77. Cutrona SL, Choudhry NK, Fischer MA, et al. Targeting cardiovascular medication adherence interventions. J Am Pharm Assoc. 2012; 52(3):381-397.
78. Gallagher R, Luttik ML, Jaarsma T. Social support and self-care in heart failure. J Cardiovasc Nurs. 2011;26(6):439-445.

79. Alosco ML, Spitznagel MB, van Dulmen M, et al. Cognitive function and treatment adherence in older adults with heart failure. Psychosom Med. 2012;74(9):965-973.

80. Garner JB. Problems of nonadherence in cardiology and proposals to improve outcomes. Am J Cardiol. 2010;105(10):1495-1501.

81. Mavrides N, Nemeroff C. Treatment of depression in cardiovascular disease. Depress Anxiety. 2013;30:328-341.

82. Joynt KE, O'Connor CM. Lessons from SADHART, ENRICHD, and other trials. Psychosom Med. 2005;67 (Suppl 1):S63-S66.

Patient Preference and Adherence

\section{Publish your work in this journal}

Patient Preference and Adherence is an international, peer-reviewed, open access journal that focuses on the growing importance of patient preference and adherence throughout the therapeutic continuum. Patient satisfaction, acceptability, quality of life, compliance, persistence and their role in developing new therapeutic modalities and compounds to optimize clinical outcomes for existing disease states are major areas of interest for the journal. This journal has been accepted for indexing on PubMed Central. The manuscript management system is completely online and includes a very quick and fair peer-review system, which is all easy to use. Visit http://www. dovepress.com/testimonials.php to read real quotes from published authors.

\footnotetext{
Submit your manuscript here: http://www.dovepress.com/patient-preference-and-adherence-journal
} 